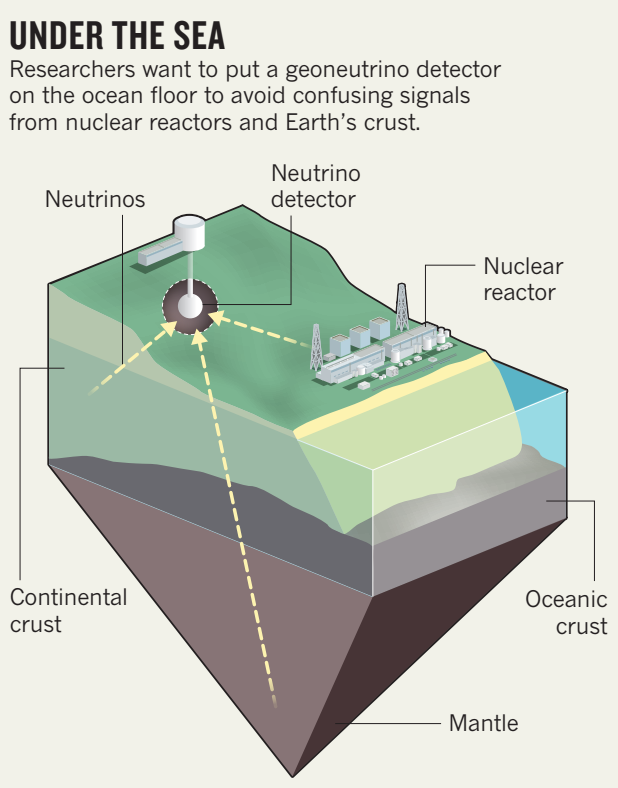

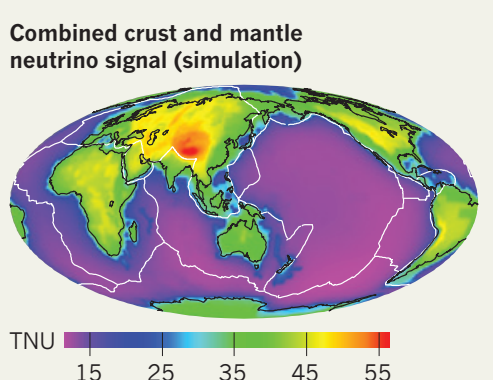

Nuclear reactor neutrino signal

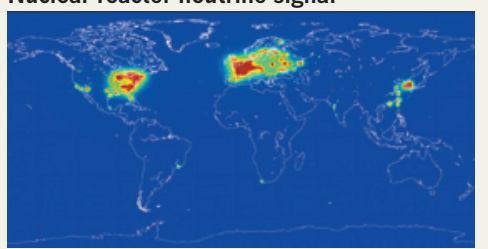

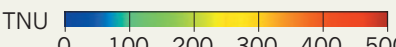

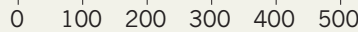

TNU: Terrestrial neutrino units. 1 TNU corresponds to 1 geoneutrino event recorded over a year-long fully efficient exposure to $10^{32}$ free protons.

\title{
Detectors zero in on Earth's heat
}

\section{Geoneutrinos paint picture of deep-mantle processes.}

\section{BY ALEXANDRA WITZE}

A window on the deep Earth opened unexpectedly in 2011, when Japan's nuclear reactors were shut down after the Fukushima disaster. Before the closure, an underground particle detector called KamLAND based in Kamioka, Japan, was monitoring a torrent of neutrinos streaming from dozens of nearby nuclear reactors, seeking clues to the nature of these hard-to-catch subatomic particles. After those plants fell silent, KamLAND scientists could see more clearly a signal that had largely been obscured: a faint trickle of neutrinos produced inside the planet.

Neutrinos are generated in stars, reactors, and deep in Earth's crust and mantle by the radioactive decay of elements such as uranium and thorium. KamLAND reported the first tentative detections of these 'geoneutrinos' in 2005 (ref. 1). But last month at a conference in Takayama, Japan, KamLAND scientists reported seeing them in meaningful quantities - as did a team at the Borexino neutrino detector at the Gran Sasso National Laboratory near L'Aquila, Italy.

These detections are not just curiosities. Geoneutrinos offer the only way to measure one of Earth's internal heat sources. The total heat flow, measured with sensors in deep mines and amounting to 47 terawatts (TW) of power, drives everything from plate tectonics to Earth's magnetic field. Some of it comes from the decay of radioactive elements, the rest is primordial heat left over from when Earth was formed by the violent collision of planetary building blocks.

But no one knows the proportions. Geologists assume that Earth contains the same amount of radioactive elements as certain primitive meteorites, but they aren't sure. "We're after trying to understand how Earth was built," says William McDonough, a geologist at the University of Maryland in College Park.

Enter KamLAND and Borexino, which spot geoneutrinos as a sideline to their other neutrino studies. Both experiments use liquid scintillator detectors, in which huge vats of fluid capture the occasional sparkle of light when a passing neutrino interacts with atomic nuclei in the liquid.

The team at Borexino, a vat containing 300 tonnes of liquid buried under the Italian Alps, captured 14 candidate geoneutrinos between December 2007 and August 2012 (ref. 2). Scientists at KamLAND, with 1,000 tonnes of liquid, say that they detected 116 probable geoneutrinos between March
2002 and November 2012 (ref. 3).

That's just enough for researchers to start drawing conclusions about the composition of Earth's mantle, says McDonough. Assuming that uranium and thorium are spread uniformly in the mantle, the KamLAND findings suggest that about 11 of the 47 TW come from the radioactive decay of those elements. A similar calculation for Borexino yields about $18 \mathrm{TW}$.

Ultimately, geoneutrino researchers would like multiple detectors spaced around Earth, so that they could perform a sort of tomography on the mantle. That could help scientists to discern between models that favour the uranium and thorium being spread throughout the mantle, versus those in which the elements are concentrated near the core-mantle boundary. Such a difference could help to determine where and how long heat will continue to flow to drive geological processes such as plate tectonics - and how long it will take Earth to cool.

One challenge is that emissions from uranium and thorium much nearer the surface in the continental crust can mask the geoneutrino signal coming from deeper in the planet (see 'Under the sea'). Next year, for example, the retrofitted Sudbury Neutrino Observatory (SNO) in Ontario, Canada, will start taking data with a 780-tonne detector that is sensitive to geoneutrinos. But $\mathrm{SNO}+$, as the upgrade is called, sits smack in the middle of continental crust. Separating crustal from mantle geoneutrinos is crucial, says Steve Dye, a physicist at Hawaii Pacific University in Honolulu, as "the mantle is really what contributes to the rate of cooling of the planet".

Dye and others say that the best way to catch mantle geoneutrinos would be from the ocean floor, where the crust is thinner than on land. One scheme, dubbed Hanohano, would lower a 10,000-tonne detector from a barge, and has been on the drawing board for years. Construction alone would cost some US $\$ 50$ million to $\$ 60$ million, says John Learned, a neutrino physicist at the University of Hawaii at Manoa in Honolulu, and the technology is ambitious.

"We've never done anything like this before," he says. But interest in the project is growing, he adds, and supporters are trying to drum up funds to keep it moving.

Meanwhile, China is working on its Daya Bay II experiment, a 20,000-tonne detector on land that could be ready to hunt for geoneutrinos in 2019. Borexino has funds to run for at least another four years. And KamLAND plans to keep going for at least five more years, says team member Hiroko Watanabe of Tohoku University in Sendai, Japan. Even after Japan's nuclear reactors restart, the detector will still be able to find geoneutrinos - just not as easily.

1. Araki, T. et al. Nature 436, 499-503 (2005)

2. Bellini, G. et al. Preprint at http://arxiv.org/ abs/1303.2571 (2013).

3. The KamLAND Collaboration. Preprint at http:// arxiv.org/abs/1303.4667 (2013). 